KUCP-0061/93

May, 1993

\title{
INTEGRABLE HIERARCHY UNDERLYING TOPOLOGICAL LANDAU-GINZBURG MODELS OF D-TYPE
}

\author{
KANEHISA TAKASAKI \\ Department of Fundamental Sciences \\ Faculty of Integrated Human Studies, Kyoto University \\ Yoshida-Nihonmatsu-cho, Sakyo-ku, Kyoto 606, Japan \\ E-mail: takasaki@jpnyitp(Bitnet)
}

\begin{abstract}
A universal integrable hierarchy underlying topological Landau-Ginzburg models of D-type is presented. Like the dispersionless Toda hierarchy, the new hierarchy has two distinct ("positive" and "negative") set of flows. Special solutions corresponding to topological Landau-Ginzburg models of D-type are characterized by a Riemann-Hilbert problem, which can be converted into a generalized hodograph transformation. This construction gives an embedding of the finite dimensional small phase space of these models into the full space of flows of this hierarchy. One of flat coordinates in the small phase space turns out to be identical to the first "negative" time variable of the hierarchy, whereas the others belong to the "positive" flows.
\end{abstract}




\section{INTRODUCTION}

Recent progress in Landau-Ginzburg models of topological strings [1] has revealed an unexpected link with theories of singularities and integrable hierarchies. In the absence of gravitational couplings (i.e., in the so called "small phase space"), these models of topological strings are reduced to a class of topological conformal field theories. Sophisticated mathematical concepts in singularity theory, such as "flat coordinates," "higher residue pairings" and "Gauss-Manin systems "[2] are now recognized as very useful tools for studying this class of topological conformal field theories [3]. These tools are also recently applied to an explicit construction of "gravitational descendents" in topological strings [4]. If gravitational couplings are turned on, flows in the space of gravitational couplings obey an integrable hierarchy of "Whitham type" or "hydrodynamic type" [5]. This is a place where the theory of integrable hierarchies plays a key role.

Relation to integrable hierarchies takes a particularly simple form in topological strings of A-type and D-type [1]. The simplest and most well understood is the case of models of A-type. These models are realized as special solutions of the dispersionless generalized KdV hierarchies. Thus the dispersionless KP hierarchy [6] emerges as a universal hierarchy of these models. The second simplest case consists of models of D-type. The Landau-Ginzburg potential of D-type contains two fields, but one of them can easily be eliminated by simple Gaussian path integral. Apart from an extra term, the reduced potential in the small phase space is essentially a special case (or an "orbifold") of A-type models. Flows in the space of gravitational couplings, however, remain to be studied. Naturally, no universal integrable hierarchy has been identified.

We present in this paper a new integrable hierarchy that underlies these topological Landau-Ginzburg models of D-type. Our construction implements, from the very beginning, an infinite number of integrable flows besides those in the small phase space. These flows should be identified with flows of gravitational couplings. Actually, the universal hierarchy is comprised of two distinct sets of flows (so to 
speak, "positive" and "negative" flows) just like the dispersionless Toda hierarchy [7]. Remarkably, an exceptional coordinate " $t_{*}$ " [1] of the small phase space can be identified with the first negative flow; this clearly explains why it is exceptional.

Even apart from the relation to topological Landau-Ginzburg medels, our new hierarchy possesses in itself a number of interesting aspects as we now show below. Let us start from exhibiting general properties, then turn to topological LandauGinzburg models.

\section{LAX FORMALISM OF NEW HIERARCHY}

The new hierarchy resemble both the dispersionless Toda hierarchy [7] and the dispersionless BKP hierarchy [8], lying thus in between. (Actually, the KP hierarchy has a C-type version, CKP, besides the B-type version [9]. They turn out to have substantially the same dispersionless limit.) Its Lax representation, like the dispersionless Toda hierarchy, consists of four sets of Lax equations of the form

$$
\begin{aligned}
& \frac{\partial \mathcal{L}}{\partial t_{2 n+1}}=\left\{\mathcal{B}_{2 n+1}, \mathcal{L}\right\}, \quad \frac{\partial \mathcal{L}}{\partial \hat{t}_{2 n+1}}=\left\{\hat{\mathcal{B}}_{2 n+1}, \mathcal{L}\right\}, \\
& \frac{\partial \hat{\mathcal{L}}}{\partial t_{2 n+1}}=\left\{\mathcal{B}_{2 n+1}, \hat{\mathcal{L}}\right\}, \quad \frac{\partial \hat{\mathcal{L}}}{\partial \hat{t}_{2 n+1}}=\left\{\hat{\mathcal{B}}_{2 n+1}, \hat{\mathcal{L}}\right\}, \quad n=0,1, \ldots,
\end{aligned}
$$

describing two sets of flows in two sets of time variables $t=\left(t_{1}, t_{3}, \ldots\right)$ and $\hat{t}=$ $\left(\hat{t}_{1}, \hat{t}_{3}, \ldots\right)$. The classical counterparts of $L$-operators

$$
\begin{aligned}
& \mathcal{L}=k+\sum_{n=1}^{\infty} u_{2 n} k^{-2 n+1}, \\
& \hat{\mathcal{L}}=\sum_{n=0}^{\infty} \hat{u}_{2 n} k^{2 n+1}, \quad \hat{u}_{0} \neq 0,
\end{aligned}
$$

are Laurent series in another variable $k$. The classical counterparts of $B$-operators 
are given by

$$
\mathcal{B}_{2 n+1}=\left(\mathcal{L}^{2 n+1}\right)_{\geq 0}, \quad \hat{\mathcal{B}}_{2 n+1}=\left(\hat{\mathcal{L}}^{-2 n-1}\right)_{\leq-1}
$$

where

$$
\begin{aligned}
& \text { ( })_{\geq 0} \text { : projection onto } k^{0}, k^{1}, \ldots, \\
& (\quad)_{\leq-1} \text { : projection onto } k^{-1}, k^{-2}, \ldots
\end{aligned}
$$

Unlike the dispersionless Toda hierarchy, however, $\mathcal{L}$ and $\hat{\mathcal{L}}$ contains only odd powers of $k$. This is a reason that only "odd" time variables are permitted. Furthermore, the Poisson bracket, too, is different and given by

$$
\{A, B\}=\frac{\partial A}{\partial k} \frac{\partial B}{\partial x}-\frac{\partial A}{\partial x} \frac{\partial B}{\partial k}, \quad x=t_{1}
$$

(The Poisson bracket in the dispersionless Toda hierarchy is defined for a rescaled lattice coordinate $s$ and its canonical momentum $p$ ) These characteristics are rather reminiscent of the dispersionless BKP (or CKP) hierarchy. In fact, the first set of Lax equations, including $\mathcal{L}$ and $t$ only, is nothing but the dispersionless BKP hierarchy. The new hierarchy is thus a straightforward extension of the dispersionless BKP hierarchy by the "negative" flows $\hat{t}$.

As in other dispersionless integrable hierarchies, one can now introduce another set of Laurent series

$$
\begin{aligned}
& \mathcal{M}=\sum_{n=0}^{\infty}(2 n+1) t_{2 n+1} \mathcal{L}^{2 n}+\sum_{n=0}^{\infty} v_{2 n+2} \mathcal{L}^{-2 n-2}, \\
& \hat{\mathcal{M}}=-\sum_{n=0}^{\infty}(2 n+1) \hat{t}_{2 n+1} \hat{\mathcal{L}}^{-2 n-2}+\sum_{n=0}^{\infty} \hat{v}_{2 n+2} \hat{\mathcal{L}}^{2 n}
\end{aligned}
$$

that satisfy the Lax equations

$$
\begin{aligned}
\frac{\partial \mathcal{M}}{\partial t_{2 n+1}} & =\left\{\mathcal{B}_{2 n+1}, \mathcal{M}\right\}, \quad \frac{\partial \mathcal{M}}{\partial t_{2 n+1}}=\left\{\hat{\mathcal{B}}_{2 n+1}, \mathcal{M}\right\}, \\
\frac{\partial \hat{\mathcal{M}}}{\partial t_{2 n+1}} & =\left\{\mathcal{B}_{2 n+1}, \hat{\mathcal{M}}\right\}, \quad \frac{\partial \hat{\mathcal{M}}}{\partial \hat{t}_{2 n+1}}=\left\{\hat{\mathcal{B}}_{2 n+1}, \hat{\mathcal{M}}\right\}, \quad n=0,1, \ldots
\end{aligned}
$$


and the canonical Poisson relations

$$
\{\mathcal{L}, \mathcal{M}\}=\{\hat{\mathcal{L}}, \hat{\mathcal{M}}\}=1
$$

Note that these $\mathcal{M}$ and $\hat{\mathcal{M}}$ contain only even powers of $\mathcal{L}$ and $\hat{\mathcal{L}}$. (This definition of $\mathcal{M}$ slightly differs from our previous notations for the dispersionless BKP hierarchy [8]; the above $\mathcal{M}$ amounts to $\mathcal{M L}^{-1}$ in the notation used therein.) As already pointed out in the case of other dispersionless hierarchies and selfdual gravity [10], this kind of extended Lax formalism is a key to a Riemann-Hilbert problem method.

\section{Riemann-Hilbert Problem}

The extended Lax representation can be rewritten into the 2 -form equation

$$
d \mathcal{L} \wedge d \mathcal{M}=d \hat{\mathcal{L}} \wedge d \hat{\mathcal{M}}=\omega
$$

where

$$
\omega=\sum_{n=0}^{\infty} d \mathcal{B}_{2 n+1} \wedge d t_{2 n+1}+\sum_{n=0}^{\infty} d \hat{\mathcal{B}}_{2 n+1} \wedge d \hat{t}_{2 n+1}
$$

Note that $\omega$ includes the symplectic 2 -form in the $(k, x)=\left(k, t_{1}\right)$ space:

$$
d \mathcal{B}_{1} \wedge d t_{1}=d k \wedge d x
$$

The above 2-form equation implies that $(\mathcal{L}, \mathcal{M})$ and $(\hat{\mathcal{L}}, \hat{\mathcal{M}})$ are two sets of "Darboux coordinates" of $\omega$ and linked by a functional relation of the form ("Riemann-Hilbert problem")

$$
\hat{\mathcal{L}}=f(\mathcal{L}, \mathcal{M}), \quad \hat{\mathcal{M}}=g(\mathcal{L}, \mathcal{M})
$$

where $f(\lambda, \mu)$ and $g(\lambda, \mu)$ are subject to the symplectic condition

$$
\frac{\partial(f(\lambda, \mu), g(\lambda, \mu))}{\partial(\lambda, \mu)}=1
$$


i.e., gives a symplectic (or area-preserving) diffeomorphism. Furthermore, in view of the parity of $\mathcal{L}, \hat{\mathcal{L}}$ (odd) and $\mathcal{M}, \hat{\mathcal{M}}$ (even) under $k \rightarrow-k$, one has to impose the extra condition

$$
f(-\lambda, \mu)=-f(\lambda, \mu), \quad g(-\lambda, \mu)=g(\lambda, \mu) .
$$

The last condition, conversely, ensures that solutions of the above Riemann-Hilbert problem indeed give solutions of the hierarchy in question (as far as only "odd" time variables are retained).

\section{S-FunCtions AND FREE ENERGY}

Our goal here is to show the existence of a potential $F$ ("free energy") along with two auxiliary functions $S$ and $\hat{S}$. The construction is parallel to those of the dispersionless Toda hierarchy. A key role is played by the residue formulas

$$
\begin{array}{cl}
\frac{\partial v_{2 m+2}}{\partial t_{2 n+1}}=\operatorname{res}_{k}\left(\mathcal{L}^{2 m+1} \partial_{k} \mathcal{B}_{2 n+1}\right), & \frac{\partial v_{2 m+2}}{\partial \hat{t}_{2 n+1}}=\operatorname{res}_{k}\left(\mathcal{L}^{2 m+1} \partial_{k} \hat{\mathcal{B}}_{2 n+1}\right), \\
\frac{\partial \hat{v}_{2 m+2}}{\partial t_{2 n+1}}=\operatorname{res}_{k}\left(\hat{\mathcal{L}}^{-2 m-1} \partial_{k} \mathcal{B}_{2 n+1}\right), & \frac{\partial \hat{v}_{2 m+2}}{\partial \hat{t}_{2 n+1}}=\operatorname{res}_{k}\left(\hat{\mathcal{L}}^{-2 m-1} \partial_{k} \hat{\mathcal{B}}_{2 n+1}\right),
\end{array}
$$

where "res $k$ " means the formal residue in $k$ :

$$
\underset{k}{\operatorname{res}} \sum a_{n} k^{n}=a_{-1}
$$

Technical details for deriving these and subsequent formulas are almost the same as the case of the dispersionless KP hierarchy [11].

As a consequence of (15) and a few basic properties of the formal residue operator, one can show the relations

$$
\begin{aligned}
& \frac{\partial v_{2 m+2}}{\partial t_{2 n+1}}=\frac{\partial v_{2 n+2}}{\partial t_{2 m+1}}, \quad \frac{\partial \hat{v}_{2 m+2}}{\partial \hat{t}_{2 n+1}}=\frac{\partial \hat{v}_{2 n+2}}{\partial \hat{t}_{2 m+1}} \\
& \frac{\partial v_{2 m+2}}{\partial \hat{t}_{2 n+1}}=-\frac{\partial \hat{v}_{2 n+2}}{\partial t_{2 m+1}}
\end{aligned}
$$


This implies the existence of a potential $F$ that reproduces $v_{2 n}$ and $\hat{v}_{2 n}$ as:

$$
\frac{\partial F}{\partial t_{2 n+1}}=v_{2 n+2}, \quad \frac{\partial F}{\partial \hat{t}_{2 n+1}}=-\hat{v}_{2 n+2} .
$$

Another consequence of (15) is an explicit expression of of $\mathcal{B}_{2 n+1}$ and $\hat{\mathcal{B}}_{2 n+1}$ as Laurent series of $\mathcal{L}$ and $\hat{\mathcal{L}}$. To see this, let us consider the formal residue operators $\operatorname{res}_{\mathcal{L}}$ and $\operatorname{res}_{\hat{\mathcal{L}}}$ with respect to $\mathcal{L}$ and $\hat{\mathcal{L}}$. They are connected with res $k$ as:

$$
\begin{aligned}
& \operatorname{res}_{\mathcal{L}} \mathcal{L}^{n}=\operatorname{res}_{k} \mathcal{L}^{n} \partial_{k} \mathcal{L}=\delta_{n,-1}, \\
& \underset{\hat{\mathcal{L}}}{\operatorname{res}} \hat{\mathcal{L}}^{n}=\operatorname{res}_{k} \hat{\mathcal{L}}^{n} \partial_{k} \hat{\mathcal{L}}=\delta_{n,-1} .
\end{aligned}
$$

By use of these relations, one can derive from (15) the following formulas.

$$
\begin{aligned}
\mathcal{B}_{2 n+1} & =\mathcal{L}^{2 n+1}-\sum_{m=0}^{\infty} \frac{1}{2 m+1} \frac{\partial v_{2 m+2}}{\partial t_{2 n+1}} \mathcal{L}^{-2 m-1} \\
& =\sum_{m=0}^{\infty} \frac{1}{2 m+1} \frac{\partial \hat{v}_{2 m+2}}{\partial t_{2 n+1}} \hat{\mathcal{L}}^{2 m+1} \\
\hat{\mathcal{B}}_{2 n+1} & =-\sum_{m=0}^{\infty} \frac{1}{2 m+1} \frac{\partial v_{2 m+2}}{\partial \hat{t}_{2 n+1}} \mathcal{L}^{-2 m-1} \\
& =\hat{\mathcal{L}}^{-2 n-1}+\sum_{m=0}^{\infty} \frac{1}{2 m+1} \frac{\partial \hat{v}_{2 m+2}}{\partial \hat{t}_{2 n+1}} \hat{\mathcal{L}}^{2 m+1}
\end{aligned}
$$

In particular, one obtains two expressions of $\mathcal{B}_{1}=k$ :

$$
\mathcal{B}_{1}=\mathcal{L}+\sum_{n=1}^{\infty} f_{2 n} \mathcal{L}^{-2 n+1}=\sum_{n=0}^{\infty} \hat{f}_{2 n} \hat{\mathcal{L}}^{2 n+1}
$$

where

$$
\begin{aligned}
& f_{2 n}=-\frac{1}{2 n-1} \frac{\partial^{2} F}{\partial t_{1} \partial t_{2 n-1}} \\
& \hat{f}_{2 n}=-\frac{1}{2 n+1} \frac{\partial^{2} F}{\partial t_{1} \partial \hat{t}_{2 n+1}}
\end{aligned}
$$


Actually, these expressions of $B_{1}=k$ give an inversion of the maps $k \rightarrow \mathcal{L}$ and $k \rightarrow \hat{\mathcal{L}}$ respectively. Solving them with respect to $\mathcal{L}$ and $\hat{\mathcal{L}}$ once again, one can express $u_{2 n}$ and $\hat{u}_{2 n}$ in terms of derivatives of $F$. For instance,

$$
u_{2}=-f_{2}=\frac{\partial^{2} F}{\partial t_{1}^{2}}, \quad \hat{u}_{0}^{-1}=\hat{f}_{0}=-\frac{\partial^{2} F}{\partial t_{1} \partial \hat{t}_{1}}
$$

The above definition of $f_{2 n}$ and $\hat{f}_{2 n}$ can be rewritten

$$
\begin{aligned}
& f_{2 n}=-\frac{1}{2 n-1} \underset{k}{\operatorname{res}} \mathcal{L}^{2 n-1}, \\
& \hat{f}_{2 n}=\frac{1}{2 n+1} \underset{k}{\operatorname{res}} \hat{\mathcal{L}}^{-2 n-1} .
\end{aligned}
$$

We shall see in the next section that these quantities are directly related to flat coordinates of Landau-Ginzburg models.

Finally, let us define the $S$-functions

$$
\begin{aligned}
& S=\sum_{n=0}^{\infty} t_{2 n+1} \mathcal{L}^{2 n+1}-\sum_{n=0}^{\infty} \frac{v_{2 n+2}}{2 n+1} \mathcal{L}^{-2 n-1}, \\
& \hat{S}=\sum_{n=0}^{\infty} \hat{t}_{2 n+1} \hat{\mathcal{L}}^{-2 n-1}+\sum_{n=0}^{\infty} \frac{\hat{v}_{2 n+2}}{2 n+1} \hat{\mathcal{L}}^{2 n+1} .
\end{aligned}
$$

As a consequence of (6) and (20), they satisfy the 1-form equations

$$
\begin{aligned}
& d S=\mathcal{M} d \mathcal{L}+\sum_{n=0}^{\infty} \mathcal{B}_{2 n+1} d t_{2 n+1}+\sum_{n=0}^{\infty} \hat{\mathcal{B}}_{2 n+1} d \hat{t}_{2 n+1}, \\
& d \hat{S}=\hat{\mathcal{M}} d \hat{\mathcal{L}}+\sum_{n=0}^{\infty} \mathcal{B}_{2 n+1} d t_{2 n+1}+\sum_{n=0}^{\infty} \hat{\mathcal{B}}_{2 n+1} d \hat{t}_{2 n+1} .
\end{aligned}
$$

Exterior differentiation of these equations reproduces 2-form equation (9). 


\section{Topological LAndaU-GinzBurg MOdels}

The $\mathrm{D}_{N}$ Landau-Ginzburg potential is a polynomial of two fields $X$ and $Y$ :

$$
W(X, Y)=\frac{X^{N-1}}{2 N-2}+\frac{1}{2} X Y^{2}+\sum_{n=1}^{N-1} g_{2 n} X^{N-n-1}+t_{*} Y
$$

where $g_{2}, \ldots, g_{2 N-2}$ depend on $N-1$ deformation variables $\left(t_{1}, t_{3}, \ldots, t_{2 N-3}\right)$; $t_{*}$ is the last deformation variable. (Our notation slightly differs from physicists' convention. Our $t_{1}, \ldots, t_{2 N-3}$ correspond to physicists' $t_{0}, \ldots, t_{2 N-4}$, up to suitable rescaling.) As pointed out by Dijkgraaf et al. [1], the field $Y$ can be eliminated by Gaussian path integral or, equivalently, by solving the relation $\partial_{Y} W(X, Y)=0$. Upon substituting

$$
X=Z^{2}
$$

the reduced potential is given by

$$
W_{\text {red }}(Z)=\frac{Z^{2 N-2}}{2 N-2}+\sum_{n=1}^{N-1} g_{2 n} Z^{2 N-2 n-2}+g_{2 N} Z^{-2}
$$

where

$$
g_{2 N}=-\frac{1}{2} t_{*}^{2}
$$

The reduced potential consists of two parts,

$$
W_{\text {red }}(Z)=W_{0}(Z)+g_{2 N} Z^{-2}
$$

the first part $W_{0}(Z)$ being interpreted as a subfamily of deformations of the $\mathrm{A}_{2 N-3}$ Landau-Ginzburg potential. The first $N-1$ deformation variables $\left(t_{1}, \ldots, t_{2 N-1}\right)$ are identical, up to rescaling by constants, to the corresponding $\mathrm{A}_{2 N-3}$ flat coordinates. The status of $t_{*}$ remains rather obscure in the work of Dijkgraaf et al. 
We now show how to interpret the reduced Landau-Ginzburg model $W_{\text {red }}(Z)$ as a special solution of our hierarchy. A very similar interpretation is recently presented by Eguchi et al. [12], however their treatment is limited to the small phase space. Our construction of solution is a generalization of our previous treatment of A-type models [11], and can cover all $(t, \hat{t})$ flows simultaneously. A RiemannHilbert problem lies in the heart of this construction.

The Riemann-Hilbert problem in this case takes the following form.

$$
\frac{\mathcal{L}^{2 N-2}}{2 N-2}=\frac{\hat{\mathcal{L}}^{-2}}{-2} \quad(\underset{\text { def }}{=} \mathcal{P}), \quad \mathcal{M} \mathcal{L}^{-2 N+3}=\hat{\mathcal{M}} \hat{\mathcal{L}}^{3} \quad(\underset{\text { def }}{=} \mathcal{Q})
$$

This Riemann-Hilbert problem does not take the standard form of (12), but the essence is the same. Note that the maps $(\mathcal{L}, \mathcal{M}) \rightarrow(\mathcal{P}, \mathcal{Q})$ and $(\hat{\mathcal{L}}, \hat{\mathcal{M}}) \rightarrow(\mathcal{P}, \mathcal{Q})$ are both symplectic. Therefore, if one can solve the above Riemann-Hilbert problem, the four functions $\mathcal{L}, \mathcal{M}, \hat{\mathcal{L}}, \hat{\mathcal{M}}$ satisfy the relation

$$
d \mathcal{L} \wedge d \mathcal{M}=d \mathcal{P} \wedge d \mathcal{Q}=d \hat{\mathcal{L}} \wedge d \hat{\mathcal{M}}
$$

which implies that they give a solution of the hierarchy.

The above Riemann-Hilbert problem, like the case of A-type models, can be reduced to a set of equations whose solvability is ensured by the implicit function theorem. The first equation of (31) simply means that $\mathcal{P}$ is an even Laurent polynomial of $k$ of the form

$$
\mathcal{P}=\frac{k^{2 N-2}}{2 N-2}+\sum_{n=1}^{N} g_{2 n} k^{2 N-2 n-2} .
$$

The coefficients of $\mathcal{P}, \mathcal{L}$ and $\hat{\mathcal{L}}$ obey algebraic relations of the form

$$
\begin{aligned}
& g_{2}=u_{2}, \quad g_{4}=u_{4}+\frac{2 N-3}{2} u_{2}^{2}, \ldots, \\
& g_{2 N}=-\frac{1}{2} \hat{u}_{0}^{-2}, \quad g_{2 N-2}=\hat{u}_{0}^{-3} \hat{u}_{2}, \ldots,
\end{aligned}
$$


which can uniquely be solved for $u_{2 n}$ and $\hat{u}_{2 n}$. The second equation of (31) requires technically more involved calculations. We first separate it into the ()$_{\leq-1 \text {-part }}$ and the ()$_{\geq 0}$-part. This results in the following equations.

$$
\begin{aligned}
& \sum_{n=0}^{N-2} t_{2 n+1} \mathcal{L}^{2 n-2 N+3}+\sum_{n=1}^{\infty} v_{2 n} \mathcal{L}^{-2 n-2 N+3} \\
= & -\sum_{n=N-1}^{\infty}(2 n+1) t_{2 n+1}\left(\mathcal{L}^{2 n-2 N+3}\right)_{\leq-1}-\sum_{n=1}^{\infty}(2 n+1) \hat{t}_{2 n+1}\left(\hat{\mathcal{L}}^{-2 n+1}\right)_{\leq-1}(35 \mathrm{a}) \\
& -\hat{t}_{1} \hat{\mathcal{L}}+\sum_{n=1}^{\infty} \hat{v}_{2 n} \hat{\mathcal{L}}^{2 n+1} \\
= & -\sum_{n=N-1}^{\infty}(2 n+1) t_{2 n+1}\left(\mathcal{L}^{2 n-2 N+3}\right)_{\geq 0}-\sum_{n=1}^{\infty}(2 n+1) \hat{t}_{2 n+1}\left(\hat{\mathcal{L}}^{-2 n+1}\right)_{\geq 0} . \quad(35 \mathrm{~b})
\end{aligned}
$$

By means of formal residue calculus, we can rewrite these equations into equations of Laurent coefficients. Eq. (35a) can thus be decomposed into

$$
\begin{aligned}
(2 n+1) t_{2 n+1}= & -\sum_{m=N-1}^{\infty}(2 m+1) t_{2 m+1} \underset{k}{\operatorname{res}}\left[\left(\mathcal{L}^{2 m-2 N+3}\right)_{\leq-1} \mathcal{L}^{-2 n+2 N-4} \partial_{k} \mathcal{L}\right] \\
& -\sum_{m=1}^{\infty}(2 m+1) \hat{t}_{2 m+1} \underset{k}{\operatorname{res}}\left[\left(\hat{\mathcal{L}}^{-2 m+1}\right)_{\leq-1} \mathcal{L}^{-2 n+2 N-4} \partial_{k} \mathcal{L}\right]
\end{aligned}
$$

for $n=0,1, \ldots, N-2$, and

$$
\begin{aligned}
v_{2 n}= & -\sum_{m=N-1}^{\infty}(2 m+1) t_{2 m+1} \underset{k}{\operatorname{res}}\left[\left(\mathcal{L}^{2 m-2 N+3}\right)_{\leq-1} \mathcal{L}^{2 n+2 N-4} \partial_{k} \mathcal{L}\right] \\
& -\sum_{m=1}^{\infty}(2 m+1) \hat{t}_{2 m+1} \underset{k}{\operatorname{res}}\left[\left(\hat{\mathcal{L}}^{-2 m+1}\right)_{\leq-1} \mathcal{L}^{2 n+2 N-4} \partial_{k} \mathcal{L}\right]
\end{aligned}
$$

for $n=1,2, \ldots$ Eq. (35b) can, similarly, be decomposed into

$$
\begin{aligned}
\hat{t}_{1}= & -\sum_{m=N-1}^{\infty}(2 m+1) t_{2 m+1} \underset{k}{\operatorname{res}}\left[\left(\mathcal{L}^{2 m-2 N+3}\right)_{\geq 0} \hat{\mathcal{L}}^{-2} \partial_{k} \hat{\mathcal{L}}\right] \\
& -\sum_{m=1}^{\infty}(2 m+1) \hat{t}_{2 m+1} \underset{k}{\operatorname{res}}\left[\left(\hat{\mathcal{L}}^{-2 m+1}\right)_{\geq 0} \hat{\mathcal{L}}^{-2} \partial_{k} \hat{\mathcal{L}}\right]
\end{aligned}
$$


and

$$
\begin{aligned}
\hat{v}_{2 n}= & \sum_{m=N-1}^{\infty}(2 m+1) t_{2 m+1} \underset{k}{\operatorname{res}}\left[\left(\mathcal{L}^{2 m-2 N+3}\right)_{\geq 0} \hat{\mathcal{L}}^{-2 n-2} \partial_{k} \hat{\mathcal{L}}\right] \\
& +\sum_{m=1}^{\infty}(2 m+1) \hat{t}_{2 m+1} \underset{k}{\operatorname{res}}\left[\left(\hat{\mathcal{L}}^{-2 m+1}\right)_{\geq 0} \hat{\mathcal{L}}^{-2 n-2} \partial_{k} \hat{\mathcal{L}}\right]
\end{aligned}
$$

for $n=1,2, \ldots$ These equations give a D-type version of the generalized hodograph transformation of A-type models [11]. The first half, (36) and (38), of these equations are to determine $g_{2}, \ldots, g_{2 N}$ (hence $\mathcal{L}$ and $\hat{\mathcal{L}}$, too) as implicit functions of $t$ and $\hat{t}$. The implicit function theorem indeed ensures the existence of a unique solution in, for instance, a neighborhood of the submanifold

$$
\begin{aligned}
& t_{2 N-1}=-\frac{1}{2 N-1}, \quad t_{2 N+1}=t_{2 N+3}=\cdots=0 \\
& \hat{t}_{3}=\hat{t}_{5}=\cdots=0 .
\end{aligned}
$$

The rest of Eqs. (37) and (39), give a considerably complicated, but explicit expression of $v_{2 n}$ and $\hat{v}_{2 n}$.

It is exactly on this submanifold, (40), that the reduced Landau-Ginzburg potential $W_{\text {red }}(Z)$ is reproduced upon identifying $Z=k$ and $W_{\text {red }}(Z)=\mathcal{P}$. In other words, this submanifold is nothing but the small phase space of the $\mathrm{D}_{N}$ model. To see this, note that Eqs. (36) and (38) are simplified on this submanifold as:

$$
\begin{aligned}
& (2 n+1) t_{2 n+1}=-f_{2 N-2 n-2} \quad(n=0, \ldots, N-2), \\
& \hat{t}_{1}=\hat{f}_{0}=\hat{u}_{0}^{-1} .
\end{aligned}
$$

The first relation tells that the $t$ variables coincide, up to rescaling constants, with flat coordinates of the $\mathrm{A}_{2 N-3}$ model restricted to the subspace $t_{2 n}=0$, thus reproducing the result of Dijkgraaf et al. [1]. The second relation, meanwhile, shows that

$$
t_{*}=\hat{t}_{1} .
$$


[Compare the two expressions of $g_{2 N}$ in (29) and (34). In view of the above relation between $\hat{t}_{1}$ and $\hat{u}_{0}$, one can readily derive (42).] Thus, the somewhat distinct deformation variable $t_{*}$ turns out to be identical to the first "negative" time of our hierarchy.

Let us finally note that the above construction can be extended straightforward to a more general case (with two discrete parameters $N$ and $\hat{N}$ ) of the form

$$
\frac{\mathcal{L}^{2 N-2}}{2 N-2}=\frac{\hat{\mathcal{L}}^{-2 \hat{N}+2}}{-2 \hat{N}+2}, \quad \mathcal{M} \mathcal{L}^{-2 N+3}=\hat{\mathcal{M}} \hat{\mathcal{L}}^{2 \hat{N}-1}
$$

In view of the general classification program of topological conformal field theories by Krichever and Dubrovin [5], these solutions, too, deserve to be studied from a physical point of view.

\section{Conclusion}

Our results are divided into two parts. In the first part, the new hierarchy is introduced and general properties are specified. The hierarchy itself, in one hand, resembles the dispersionless Toda hierarchy in the sense that it consists of two distinct sets of flows. The "positive" flows, on the other hand, are substantially the same as the dispersionless BKP (or CKP) hierarchy. In the second part, topological Landau-Ginzburg models of D-types are identified with special solutions of this hierarchy. These solutions are characterized by a Riemann-Hilbert problem, which can be converted into a generalized hodograph transformation.

Several new aspects of D-type models are revealed in these results. For instance, one can clarify the status of the somewhat distinct deformation variable $t_{*}$ in these models. In fact, $t_{*}$ turns out to be identical to the first "negative" time variable of the hierarchy, whereas the other variables $t_{1}, \ldots, t_{2 N-3}$ belong to the "positive" flows. The finite dimensional small phase space with these "flat coordinates" are now embedded into the full space of flows of the hierarchy. The time variables other than $t_{1}, \ldots, t_{2 N-3}$ and $\hat{t}_{1}$ should be interpreted as gravitational 
couplings. Furthermore, although lacking physical interpretation, more general solutions with two discrete parameters are shown to be constructed in the same manner.

We add a few comments.

1. As already pointed out by Dijkgraaf et al. [1], topological Landau-Ginzburg models of D-type are related to Drinfeld-Sokolov hierarchies of D-type. The hierarchy of $\left(D_{N}^{(1)}, c_{0}\right)$ type in their table, indeed, turns out to reproduce the $t$ flows of $W_{\text {red }}(Z)$ (or of $\mathcal{P}$ ) in dispersionless (or quasi-classical) limit. Deriving the $\hat{t}$ flows remains to be solved. This issue seems to require a suitable extension of the Drinfeld-Sokolov theory.

2. An infinite dimensional Lie algebra called $D_{\infty}$ and an associated integrable hierarchy are presented by Jimbo and Miwa [13]. This is a D-type version of the KP hierarchy. The $D_{N}^{(1)}$ algebras are included in $D_{\infty}$ as subalgebras, and associated reductions are shown (at least at the level of Hirota bilinear equations) to agree with the Drinfeld-Sokolov $\left(D_{N}^{(1)}, c_{0}\right)$ hierarchies. Furthermore, remarkably, the hierarchy consists of two sets of flows like the $(t, \hat{t})$ flows of our hierarchy. Thus, the hierarchy of Jimbo and Miwa will presumably be a "dispersionful" counterpart of our hierarchy.

The author would like to thank Takashi Takebe for many useful comments. He is also grateful to Dr. Yasuhiko Yamada for hospitality at a KEK seminar on topological field theories and period integrals. This work is partly supported by the Grant-in-Aid for Scientific Research, the Ministry of Education, Science and Culture, Japan. 


\section{REFERENCES}

1. Dijkgraaf, R., Verlinde, E., and Verlinde, H., Topological strings in $d<1$, Nucl. Phys. B352 (1991), 59-86.

2. K. Saito, On the period of primitive integrals, Publ. RIMS, Kyoto Univ., 19 (1983), 1231.

M. Noumi, Expansion of the solutions of a Gauss-Manin system at a point of infinity, Tokyo J. Math. 7 (1984), 1-60.

3. Blok, B., and Varchenko, A., Topological conformal field theories and the flat coordinates, Int. J. Mod. Phys. A7 (1992), 1467-1490.

4. Losev, A., Descendents constructed from matter field in topological LandauGinzburg theories to topological gravity, ITEP preprint (November, 1992). Eguchi, T., Kanno, H., Yamada, Y., and Yang, S.-K., Topological strings, flat coordinates and gravitational descendents, University of Tokyo preprint UT-630 (February, 1993).

5. Krichever, I.M., The dispersionless Lax equations and topological minimal models, Commun. Math. Phys. 143 (1991), 415-426.

Dubrovin, B.A., Hamiltonian formalism of Whitham-type hierarchies and topological Landau-Ginsburg models, Commun. Math. Phys. 145 (1992), 195-207.

6. Lebedev, D., and Manin, Yu., Conservation laws and Lax representation of Benny's long wave equations, Phys.Lett. $74 \mathrm{~A}$ (1979), 154-156.

Kodama, Y., A method for solving the dispersionless KP equation and its exact solutions, Phys. Lett. 129A (1988), 223-226.

Kodama, Y., and Gibbons, J., A method for solving the dispersionless KP hierarchy and its exact solutions, II, Phys. Lett. 135A (1989), 167-170.

7. Takasaki, K., and Takebe, T., SDiff(2) Toda equation - hierarchy, tau function and symmetries, Lett. Math. Phys. 23 (1991), 205-214. 
8. Takasaki, K., Quasi-classical limit of BKP hierarchy and W-infinity symmetries, Kyoto University preprint KUCP-0058/93 (January, 1993).

9. Date, E., Jimbo, M., Kashiwara, M., and Miwa, T., Transformation theory for soliton equations IV, J. Phys. Soc. Japan 50 (1982), 3813-3818.

10. Takasaki, K., Area-preserving diffeomorphisms and nonlinear integrable systems, in: Topological and geometrical methods in field theory, Turku, Finland, 1991, ed. J. Mickelsson and O. Pekonen (World Scientific, Singapore, 1992).

11. Takasaki, K., and Takebe, T., SDiff(2) KP hierarchy, in: Infinite Analysis, RIMS Research Project 1991, Int. J. Mod. Phys. A7, Suppl. 1 (World Scientific, Singapore, 1992).

12. Eguchi, T., Yamada, Y., and Yang, S.-K., Topological field theories and the period integrals, University of Tokyo preprint UT-641 (April, 1993).

13. Jimbo, M., and Miwa, T., Solitons and infinite dimensional Lie algebras, Publ. RIMS, Kyoto Univ., 19 (1983), 943-1001. 